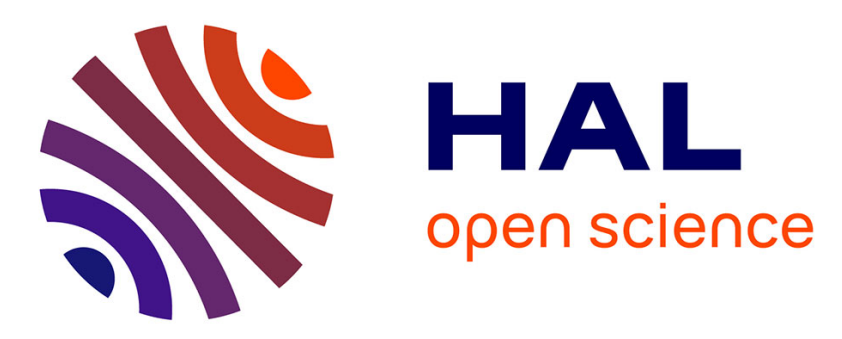

\title{
Analysis of frequency response and noise of CCD structures
}

S. Donati, F. Montecchi

\section{To cite this version:}

S. Donati, F. Montecchi. Analysis of frequency response and noise of CCD structures. Revue de Physique Appliquée, 1978, 13 (12), pp.691-696. 10.1051/rphysap:019780013012069100 . jpa00244527

\section{HAL Id: jpa-00244527 https://hal.science/jpa-00244527}

Submitted on 1 Jan 1978

HAL is a multi-disciplinary open access archive for the deposit and dissemination of scientific research documents, whether they are published or not. The documents may come from teaching and research institutions in France or abroad, or from public or private research centers.
L'archive ouverte pluridisciplinaire HAL, est destinée au dépôt et à la diffusion de documents scientifiques de niveau recherche, publiés ou non, émanant des établissements d'enseignement et de recherche français ou étrangers, des laboratoires publics ou privés. 


\title{
ANALYSIS OF FREQUENCY RESPONSE AND NOISE OF CCD STRUCTURES
}

\author{
S. DONATI and F. MONTECCHI
}

Istituto di Elettronica, Università di Pavia, 27100 Pavia, Italy

\begin{abstract}
Résumé. - On présente un modèle de cellules pour le CCD dans le cas des faibles signaux, qui rend compte complètement de la fonction de filtrage et du bruit donné par le dispositif. L'analyse peut être appliquée soit au registre séquentiel (analogique ou numérique), soit au CCD à image, et comprend les effets des : retard d'horloge, inefficacité du transfert, bruits du stockage et du transfert, et le Moiré dû à l'échantillonnage d'entrée.
\end{abstract}

Abstract. - A small-signal cell-model of the CCD is proposed, which is shown to account fully for frequency-response and noise performances of the device. The analysis applies both to shiftregister (analog or digital) structures and to imaging CCDs, and includes the effects of clock delay, transfer inefficiency, storage and transfer noises, and sampling-generated aliasing (Moiré).

1. Introduction. - Since its proposal in 1970 $[1,2]$, the CCD has been developed to the point of a well-established component-family. Disseminated in the areas of analog-filtering, memory and image applications, all CCD components share the common basic concept of charge storage and transfer under a MOS multielectrode structure. Therefore, it appears timely to carry out an analysis of the fundamental electrical performances of the device - namely frequency-response and noise - as limited by those basic variable relations which are implied by the CCD physical operation. Here, we will use a system-theory approach to describe the single CCD cell through a model and to derive the properties of a $n$-cell device. The approach lends itself to a new and straightforward derivation of all the relevant $C C D$ performances. New results are found in addition to previously known ones, and are reported in parametric form to allow a quick evaluation of their dependence from the design variables, both for electrical and image applications.

2. Cell model and transfer functions. - As it is well known, transfer of charge packet between adjacent cells is promoted by thermal diffusion, fringing field and self-induced drifts [3], while the finite time allowed for the transfer and the trapping of charge in fast states are responsible for the small percentage of residual charge left back after the transfer period [3]. A constant transfer inefficiency $\varepsilon$ describes these mechanisms, which are basically signal-amplitude independent and also imply that the residual charge is available for the next transfer after a clock period $T$.

Regardless the cell 2- or 3-phase arrangement, we can then model the elementary CCD-cell as in figure 1 , where the delay-block $\exp (-j \omega T)$ represents the cell delay at a clock period $T$, the fraction $1-\varepsilon$ of its output being sent directly to the next cell, and the feedback loop $\varepsilon$ represents the lost charge injection

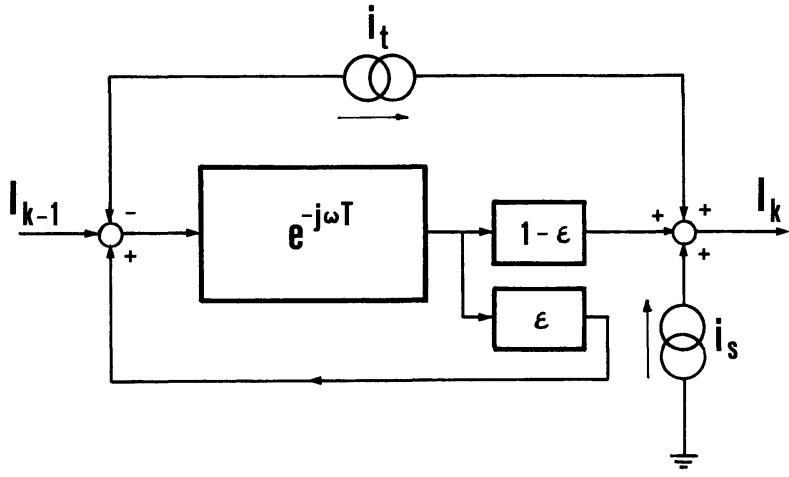

Fig. 1. - Model of the elementary CCD cell.

back at the input for the availability at the next clock period.

These blocks are sufficient to account for the deterministic response of the transfer in a CCD; the noise generators in figure 1 will be discussed later on. Indeed, we can write at once the transfer function $F(\omega)$ of the cell by applying the basic equation of a feedback loop :

$$
F(\omega)=\frac{(1-\varepsilon) \exp (-j \omega T)}{1-\varepsilon \exp (-j \omega T)}
$$

and the overall transfer function $F_{n}(\omega)$ of a CCD composed of $n$ identical cells in cascade, as :

$$
F_{n}(\omega)=F^{n}(\omega)=\frac{(1-\varepsilon)^{n} \exp (-j n \omega T)}{[1-\varepsilon \exp (-j \omega T)]^{n}} .
$$

Eq. (2) depends on both $n$ and $\varepsilon$, but a more simple and suitable expression turns out with the assumption of a large number of cells $n$. Letting in (2) the Euler's limit $(1-\mathrm{a} / n)^{n}=\exp (-a)$, we have :

$$
F_{n}(\omega)=\exp \{-n \varepsilon[1-\exp (-j \omega T)]\}
$$


and now the transfer function only depends on the inefficiency product $n \varepsilon$. Eq. (3), where the overall delay $n T$ has been omitted, was found in a different way by Buss et al. [4] and is nicely accurate : when numerical results are compared to those of eq. (2), we find that they hold within a $5 \%$ error even for moderate $n$ (e.g. $n \geqslant 5$ ) in the interesting range of $\varepsilon$ (up to $\varepsilon=0.1$ ) [5]. Similarly, also the noise expressions derived below in the Euler's limit have been found [5] accurate within the same bounds. The transfer function $F_{n}(\omega)$ given by eqs. (2) or (3) directly accounts for the charge-transfer process in a $n$-cell CCD handling electrical signals. It can also be used for an image$\mathrm{CCD}$, for which the spatial frequency response is of more significance, by noting that the cell spacing $l$ is scanned in a clock period $T$. Accordingly, a correspondence $k l=\omega T$ is settled by the device geometry, between the time-frequency $\omega$ of electrical signal and the spatial frequency $k$ of the image-line contained in it along the scanned direction. Therefore, the optical transfer function $(O T F)$ due to the charge-transfer is obtained by letting $\omega T=k l$ in eq. (3) :

$$
O T F(k)=\exp \{-n \varepsilon[1-\exp (-j k l)]\}
$$

and gives, taking its modulus, the previously known $[6,7]$ expression of the modulation transfer function $(M T F)$ in the large $n$ approximation :

$$
\operatorname{MTF}(k)=|O T F(k)|=\exp \{-n \varepsilon[(1-\cos k l)]\} .
$$

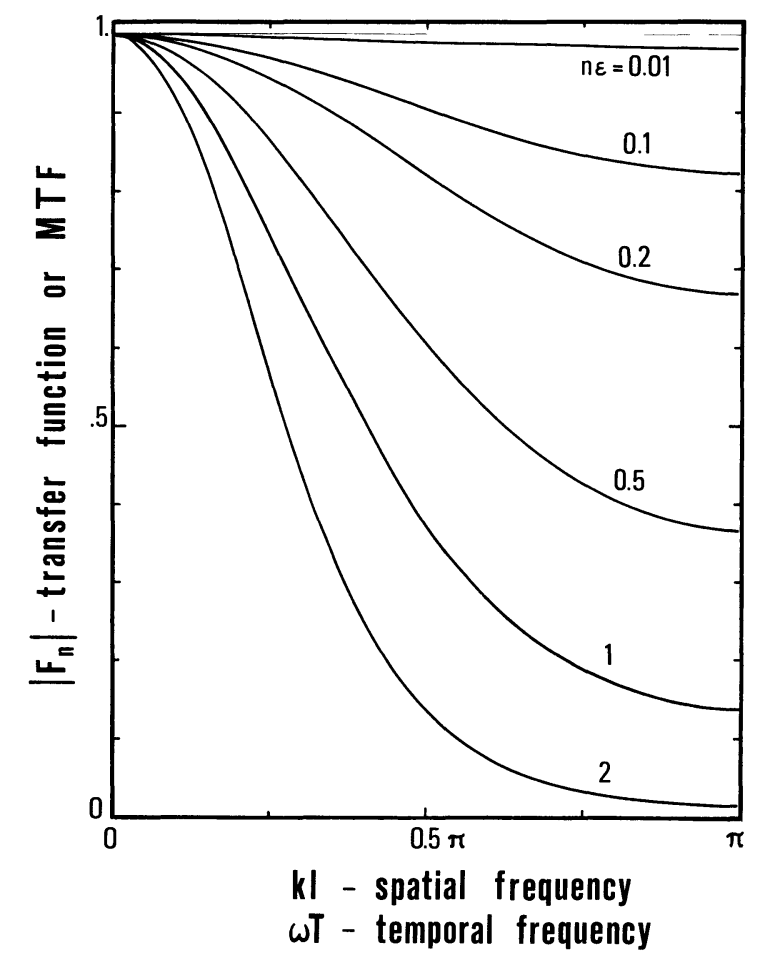

FIG. 2. - Transfer function $\left|F_{n}\right|$ or $M T F$ of a $n$-cell CCD device as a function of frequency (spatial or termporal) for some values of the inefficiency product $n \varepsilon$.
Note how, in an image-CCD, the $M T F$ degradation due to the charge-transfer depends from the point in the image, since the number of crossed cell varies with the distance of the sensor area from the collector output. Also, in an array device two spatial frequencies $k_{1}$ and $k_{2}$ are associated with the orthogonal scan directions, and eq. (5) shall be duplicated accordingly. Thus, we can see that the image supplied by the CCD would not be aplanatic if the charge-transfer degradation is the dominant one. In figure 2 it is plotted the function $\left|F_{n}\right|$ or $M T F$.

An additional contribution to the degradation of the transfer function, common to the CCDfamily, is due to the integration and sampling process by which charge is injected at the first CCD cell. This process is also responsible for the generation of spurious signals, the so-called aliasing or Moiré effect. For electrical input signals, the CCD-input diode fills of signal charge the first cell potential well during a fraction $\eta T$ of the clock period, and the subsequent transfer acts as a sampling which dumps the integrated charge. In an image-CCD, the role of input-diode is taken by the profile of photodetector cell, over which the spatial distribution of illumination is integrated, and the sampling is again provided by charge transfer [8]. Therefore, the relation between the true CCD input signal $i(t)$ and the sampled input $i_{0}(t)$ entering the CCD cells (Fig. 3 ) can be written as :

$$
i_{0}(t)=[i(t) * c(t)] \cdot \operatorname{comb}(t)
$$

where comb (.) is a sequence of $\delta$-functions at clock times $m T$ (see Fig. 3) which describes the ideal sampling, and $c(t)$ is the impulse response of the integrateand-dump section (the counterpart $c(x)$ for an image device is the cell-profile function [8]).

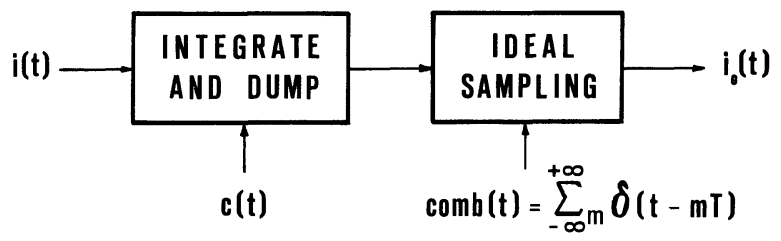

FIG. 3. - Model of the CCD-input sampling process.

In the frequency domain, eq. (6) becomes :

$$
\begin{aligned}
I_{0}(\omega) & =I(\omega) \cdot C(\omega) * \operatorname{comb}(\omega) \\
& =\sum_{-\infty}^{+\infty} I(\omega-2 \pi m / T) C(\omega-2 \pi m / T)
\end{aligned}
$$

which contains both the transfer function $(m=0)$ and aliasing $(m \neq 0)$ contributions. In fact, if the input signal is band-limited to less than the Nyquist frequency $f_{\mathrm{N}}=1 / 2 T$, eq. (7) reduces to the single $m=0$ term and a sampling transfer function $F_{\mathrm{s}}(\omega)$ can be defined because the process is stricly a linear one :

$$
F_{\mathrm{s}}(\omega)=I(\omega) / I_{0}(\omega)=C(\omega)
$$


or, $M_{T} F_{\mathrm{s}}(k)=C(k)$. The $m \neq 0$ terms in eq. (7) are those down-converted components of input signal spectrum reflected into the baseband, and they generate an aliasing signal $A(\omega)$ given by :

$A(\omega)=\sum_{-\infty}^{+\infty} I(\omega-2 \pi m / T) F_{\mathrm{s}}(\omega-2 \pi m / T)$

and similarly for $A(k)$. Now, in the schematic case of a rectangular profile of intergration,

or

$$
c(t)=1(t)-1(t-\eta T)
$$

$$
c(x)=1(x)-1(x-\eta l),
$$

filling a fractional width $\eta$ of clock-period $T$ or of cell-spacing $l$, one has :

$$
\begin{aligned}
F_{\mathrm{s}}(\omega) & =C(\omega)=\sin (\eta \omega T / 2) /(\omega T / 2) \\
M T F_{\mathrm{s}}(k) & =C(k)=\sin (\eta k l / 2) /(k l / 2)
\end{aligned}
$$

a well-known result [7,9], which is plotted in figure 4 with $\eta$ as a parameter, to show how a high inputinjection efficiency ( $\eta$ near to 1$)$ depresses the transfer function near the Nyquist frequency. This is just the case of an image CCD where $\eta$ is usually maximized and the sampling $M T F$ can override the transfer $M T F$, while in an electric-signal CCD $\eta$ is 0.5 or 0.33 for a 2 - or 3-phase clock respectively, and sampling transfer function is a minor correction to overall transfer function $F=F_{\mathrm{s}} F_{\mathrm{n}}$. An important feature again dependent from $\eta$ is the aliasing signal. The worst case is that of a white-spectrum input signal, $I=$ const., for which eq. (8) is still valid if only the original baseband content of $I$ is considered. However,

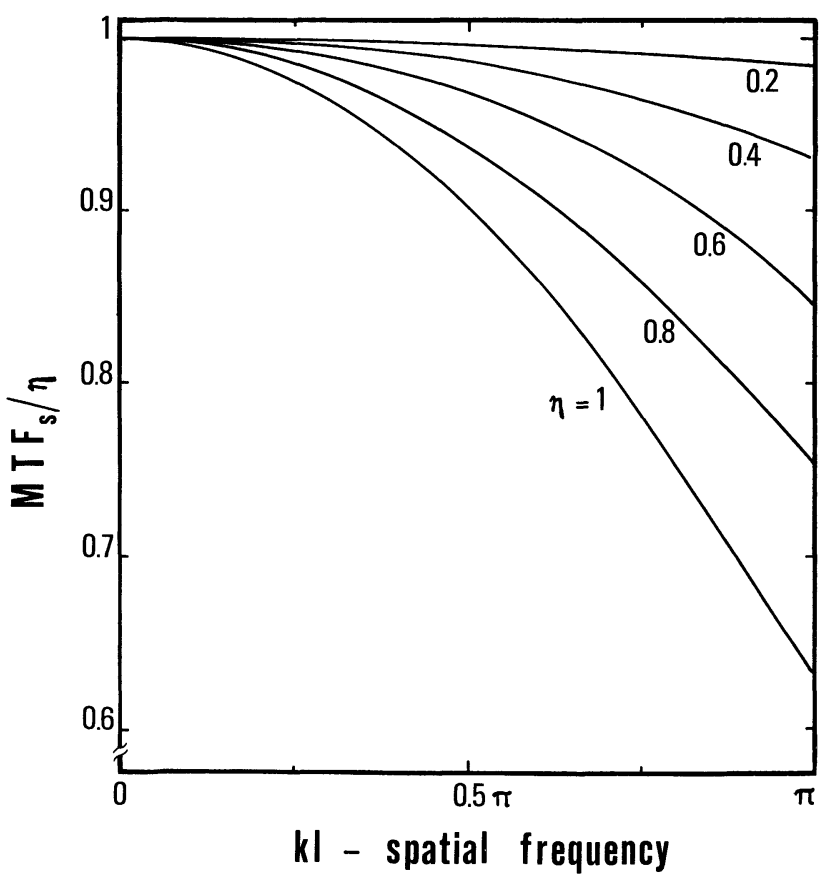

Fig. 4. - MTF or transfer function due to the CCD-input integration and sampling. other contributions are now added in the baseband, i.e. the tails of spectrum which are mirror-reflected around integer multiples of the sampling frequency $1 / T$ or $1 / l$ (eq. (9)). We can define a disturbance figure $\rho_{m}$ for the band of $m$-th order (i.e. for the band $m f_{\mathrm{N}}<f<(m+1) f_{\mathrm{N}}$, see eq. (9)) as the ratio of the output disturbance power to the power of output signal baseband-components :

$$
\rho_{m}=\int_{0}^{\pi / T} A_{m}^{2}(\omega) F_{\mathrm{n}}^{2}(\omega) \mathrm{d} \omega / \int_{0}^{\pi / T} F_{\mathrm{s}}^{2}(\omega) F_{\mathrm{n}}^{2}(\omega) \mathrm{d} \omega
$$

where $\left.A_{m}=F_{\mathrm{s}}(\omega-\pi(m+1) / T)\right)$ for $m$ odd, and $A_{m}=F_{\mathrm{s}}(\omega+\pi m / T)$ for $m$ even. With a rectangular profile for $F_{\mathrm{s}}$, as that of eq. (10), the disturbance figure $\rho_{m}$ has been calculated for $m=1 \div 4$, and has been plotted in figure 5 versus $\eta$ for two representative values of $n \varepsilon$. These diagrams point out that attempts to improve the transfer function by increasing $\eta$ may result in a poor aliasing performance, and they supply the quantitative starting data for aliasing reduction techniques $[10,11]$ based on prefiltering of the input signal, such as schemes of oversampling for the electrical CCD $[10,11]$ or objective-lens $M T F$ tailoring for the image CCD.

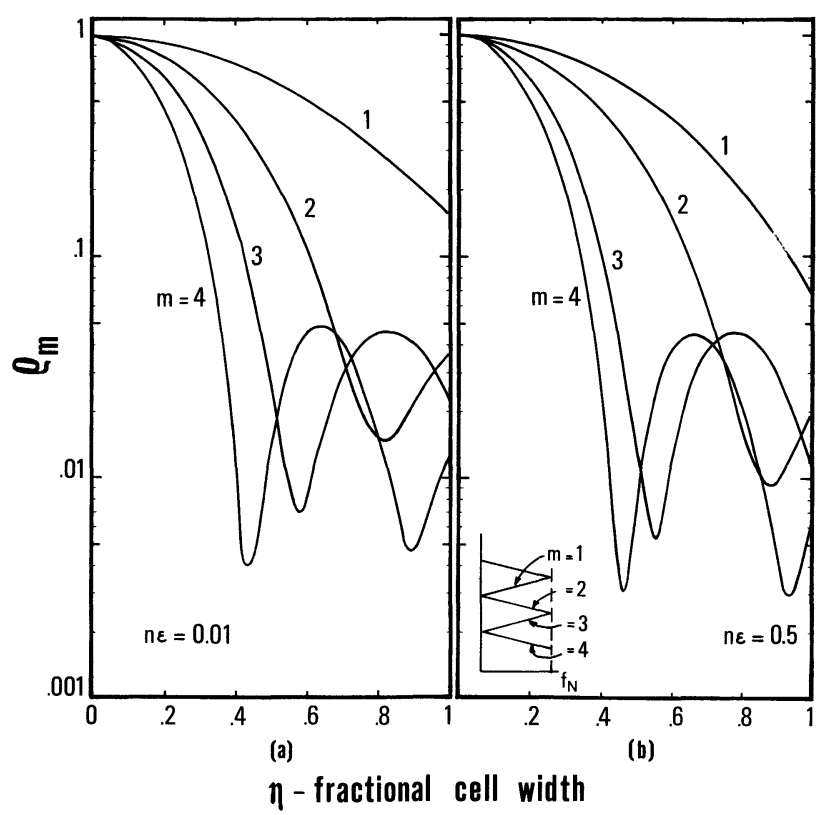

FIG. 5. - The ratio $\rho_{m}$ of aliasing and baseband signals vs the fractional width of integration $\eta$, for various intervals

$$
m f_{\mathrm{N}}<f<(m+1) f_{\mathrm{N}}
$$

at multiples of the Nyquist frequency $f_{\mathrm{N}}$.

3. Noise modelling and spectrum. - Transfer and storage of charge packets in the $\mathrm{CCD}$ have been recognized as the most important inherent sources of CCD noise. Unlike charge injection noise at the 
input and charge spilling noise at the output, which are essentially thermally limited processes and give the basic $k T / C$ charge fluctuation, transfer and storage noises are peculiar sources of CCDs with no counterpart in other electronic devices.

To model these noises, let us refer explicitly to the time domain variables. All subsequent expressions are also valid in the space domain with the correspondence $\omega T=k l$. In the previous section, the average effects of incomplete charge transfer were accounted for by a constant inefficiency $\varepsilon$ used in the blocks of figure 1. In both phenomena of trapping in fast states and of insufficient time allowed to transfer, there are associated charge fluctuations which are clearly anticorrelated over subsequent clock periods. Indeed, traps are filled at the beginning of a clock period by the incoming charge and their possible decay is followed by a fast refill, except during the finite transfer time when carrier concentration is low and the charge released by traps adds a fluctuation to the transferred charge that will be balanced in the next clock period by the incoming charge packet [9, 12]. Thus, these fluctuations can be described in the cell model by a noise generator $i_{\mathrm{t}}$ placed accross the cell, so that at the cell input the injected fluctuation is opposite to that injected to the output. Other charge fluctuation contributions come from generationrecombination during the storage period and from slow trapping states [9], the events of which are randomly distributed in time, i.e. uncorrelated to the clock or signal waveforms. Therefore, we model this storage noise by a generator $i_{\mathrm{s}}$ placed at the output node of the cell (Fig. 1). Obviously, the first and last generator could include also input/output noises or the shot-noise of detected quanta for an image CCD, contributions that we do not treat in the following.

We can now assume statistical independence for noise generators $i_{\mathrm{t}}$ and $i_{\mathrm{s}}$ and for generators of different cells. A viable representation for them is a sequence of $\delta$-pulses at clock frequency with random charge $q$. If $\left\langle q^{2}\right\rangle-\langle q\rangle^{2}=q_{\mathrm{t}}^{2}$ or $q_{\mathrm{s}}^{2}$ is the variance of the random charge assumed equal for all cells, the power spectral densities $S_{\mathrm{t}}(\omega)$ and $S_{\mathrm{s}}(\omega)$ of transfer and storage generators are given by [5] :

$$
S_{\mathrm{t}}(\omega)=q_{\mathrm{t}}^{2} / T, \quad S_{\mathrm{s}}(\omega)=q_{\mathrm{s}}^{2} / T
$$

From figure 1 we can see that the $k$-th cell noise contribution $i_{k}$ due to $i_{\mathrm{t}}$ and $i_{\mathrm{s}}$ is :

$$
i_{k}=i_{\mathrm{s}}+i_{\mathrm{t}}(1-F)
$$

where $F$ is given by eq. (1). The corresponding spectral density $S_{k}$ is therefore :

$$
S_{k}=q_{\mathrm{s}}^{2} / T+\left(q_{\mathrm{t}}^{2} / T\right)|1-F|^{2}
$$

and by summation over the $n$-cell contributions, each weighted by the proper transfer function to the output, we obtain for the bilateral power spectral density $S_{n}$ of the output noise :

$$
\begin{aligned}
S_{n} & =\sum_{1}^{n}|F|^{2(n-k)} S_{k} \\
& =\frac{q_{\mathrm{s}}^{2}}{T} \frac{1-|F|^{2 n}}{1-|F|^{2}}+\frac{q_{\mathrm{t}}^{2}}{T}|1-F|^{2} \frac{1-|F|^{2 n}}{1-|F|^{2}} \\
& =\left[\frac{q_{\mathrm{s}}^{2}}{T}\left(1+\frac{1-\varepsilon^{2}}{2 \varepsilon(1-\cos \omega T)}\right)+\frac{q_{\mathrm{t}}^{2}}{T \varepsilon}\right]\left[1-\frac{(1-\varepsilon)^{2 n}}{\left(1+\varepsilon^{2}-2 \varepsilon \cos \omega T\right)^{n}}\right] .
\end{aligned}
$$

As above, a simpler and accurate expression can be found from eq. (14) in the large $n$ approximation, i.e. :

$$
S_{n}=\left[\frac{q_{\mathrm{s}}^{2}}{T}\left(1+\frac{1-\varepsilon^{2}}{2 \varepsilon(1-\cos \omega T)}\right)+\frac{q_{\mathrm{t}}^{2}}{T \varepsilon}\right]\{1-\exp [-2 n \varepsilon(1-\cos \omega T)]\} .
$$

The transfer noise dependence of eq. (15) was obtained by Buss et al. [4] with a different approach. For $n \varepsilon \ll 1$, eq. (15) becomes :

$$
\begin{aligned}
S_{n} & =\frac{q_{\mathrm{s}}^{2}}{T}\left[2 n \varepsilon(1-\cos \omega T)+n(1-\varepsilon)^{2}\right]+\frac{2 n q_{\mathrm{t}}^{2}}{T}(1-\cos \omega T) \\
& \simeq \frac{n q_{\mathrm{s}}^{2}}{T}+\frac{2 n q_{\mathrm{t}}^{2}}{T}(1-\cos \omega T)
\end{aligned}
$$

thus indicating a nearly white spectrum for the storage noise (first term) and the well-known [9] 1-cos behaviour for the transfer noise spectrum (second term). The zero-frequency suppression of the transfer noise is however a general result contained in eq. (14) or (15). The trends of transfer noise $S_{\mathrm{Nt}}$ and storage noise $S_{\mathrm{Ns}}$ are plotted against frequency in figures 6 and 7 , and show the different character of these noises 


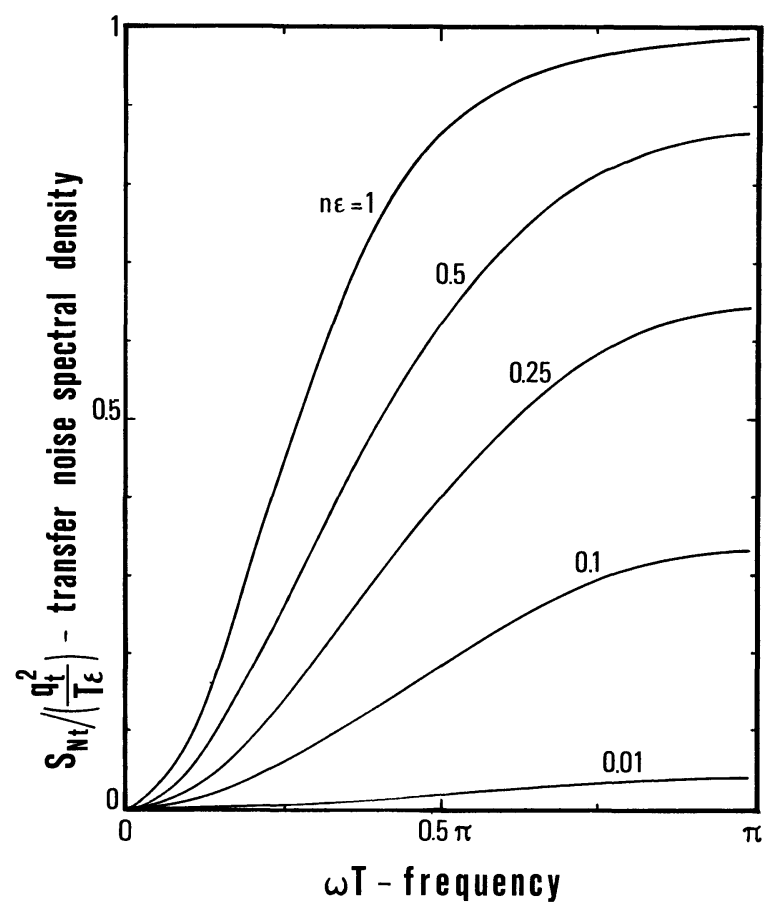

FIG. 6. - Power spectral density of transfer noise at the CCD output, normalized to $q_{\mathrm{t}}^{2} / T \varepsilon$.

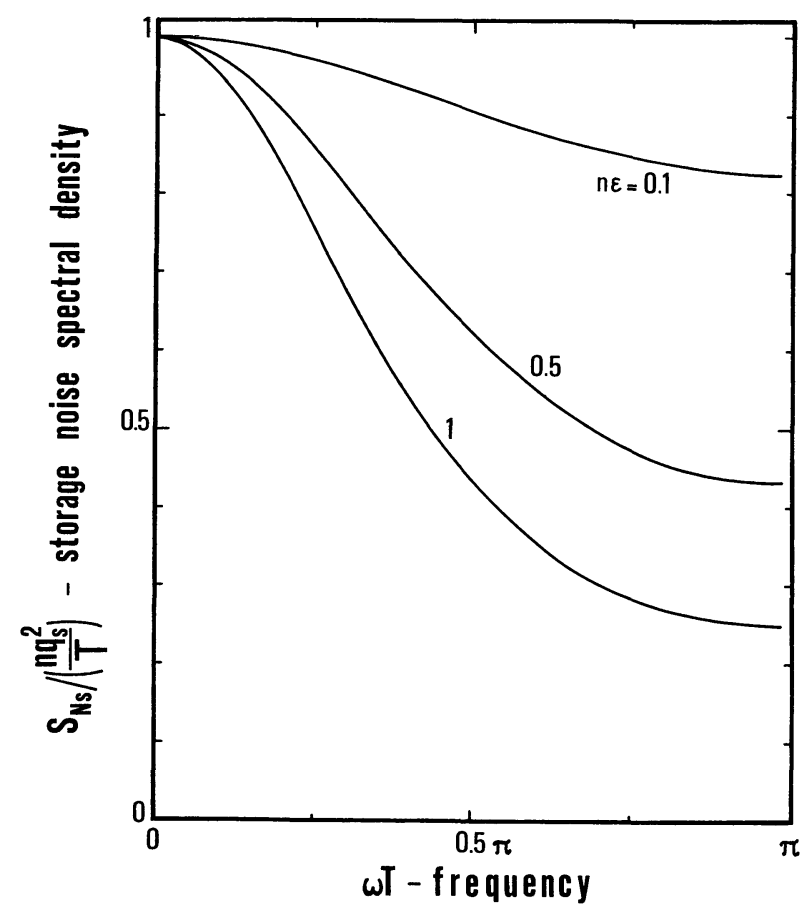

FIG. 7. - Power spectral density of storage noise at the CCD output, normalized to $n q_{\mathrm{s}}^{2} / T$.

respect to frequency and inefficiency product. A remark is now due about the tacit assumption of constant charge variances $q_{\mathrm{t}}^{2}$ and $q_{\mathrm{s}}^{2}$. For very low signals, such as in low-light-level applications of CCDs, the fast trapping states are possibly not completely filled by signal charge, and the transfer variance $q_{\mathrm{t}}^{2}$ should be taken proportional to the signal charge $q$. An analysis of the phenomena, based on the generating function approach [13], here omitted for brevity, gives as a result a multiplicative rather than additive transfer-noise, with the same expression as in eq. (15) provided $q_{\mathrm{t}}^{2} / T \varepsilon$ is changed in $q e / T$ ( $e=$ electron charge) ; thus eq. (15) is a conservative estimate even in the small signal case $q<q_{\mathrm{t}}^{2} / e \varepsilon$.

The noise dependence from $n \varepsilon$ implies again, in a CCD imager, a disuniform low-light-level performance that worsens toward the corner farthest from the output, which is actually observed (see e.g. Figs. 15 and 10 of Ref. [14]), when input/output noises are minimized.

To summarize in a single figure the noise properties of a $\mathrm{CCD}$, it is customary to introduce the noiseequivalent-signal $N E S$, defined as the input signal which would supply at the CCD output a $S / N$ ratio equal to unity under some specified operating condition. To simplify the presentation of results, we take firstly the input to which $N E S$ is calculated as the first CCD cell after the integration and sampling. For a direct use of the CCD, the output $S / N$ ratio depends on the input signal spectrum and on the bandwidth, which we will specify respectively as the class of white-spectrum signal (for the maximum generality) and as the Nyquist' band. In these conditions we have :

$$
(N E S)^{2}=\int_{-1 / 2 T}^{1 / 2 T} S_{n} \mathrm{~d} f / \int_{-1 / 2 T}^{1 / 2 T}\left|F_{n}\right|^{2} \mathrm{~d} f .
$$

Alternatively, a different and more meaningful condition is that of requiring the equalization of the CCD output, by which the output $S / N$ is independent from the input signal and the calculated $N E S$ does not improve with frequency response deterioration like it is in the direct use. In this case, limiting again the bandwidth to the maximum conceptual one, i.e. the Nyquist frequency, one has :

$$
(N E S)^{2}=\int_{-1 / 2 T}^{1 / 2 T}\left(S_{n} /\left|F_{n}\right|^{2}\right) \mathrm{d} f .
$$

In figure 8 we plot the $N E S$ calculated from eqs. (17) and (18), and standardized to the minimum noise current $\left(2 n q_{\mathrm{t}}^{2}+n q_{\mathrm{s}}^{2}\right)^{1 / 2} / T$ which would be predicted intuitively if there were no compounding [9] of the various noise contributions. Note how both direct and equalized output $N E S^{\prime}$ depart appreciably from this minimum value at about $n \varepsilon=0.5$, and that there are only minor differences between curves for $q_{\mathrm{s}}^{2} / q_{\mathrm{t}}^{2}$ ranging from 0 to 1 .

Now, moving the input node to which the $N E S$ is calculated just at the actual CCD-input, eqs. (17) and (18) shall be modified by changing $F_{n}$ in $F_{n} F_{\mathrm{s}}$, where $F_{\mathrm{s}}$ is the sampling transfer function given by eq. (8). For the rectangular cell-profile of eq. (10), 


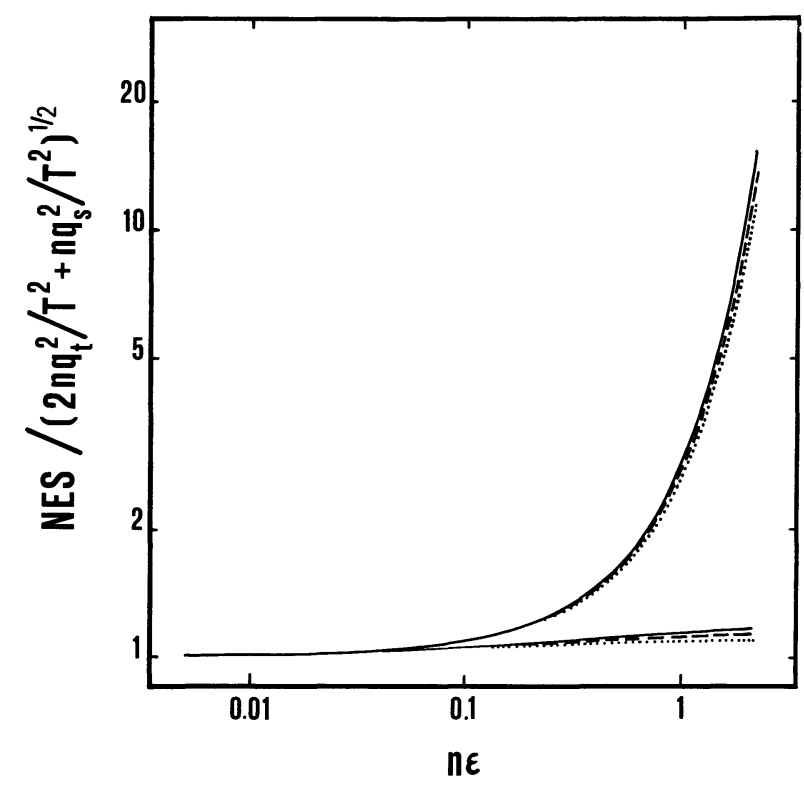

FIG. 8. - Noise equivalent signal (NES) at the first CCD cell as a function of the inefficiency product $n \varepsilon$ for a direct output (lower curves) and equalized output (upper curves). The point line is for $q_{\mathrm{s}}^{2} / q_{\mathrm{t}}^{2}=0$, the full line for $q_{\mathrm{s}}^{2} / q_{\mathrm{t}}^{2}=1$.

numerical results are found expressible with good accuracy through a single multiplicative factor $M_{\mathrm{F}}$ of the preceding $N E S$, in the range of variables plotted in figure 9 and for both the direct and equalized output conditions.

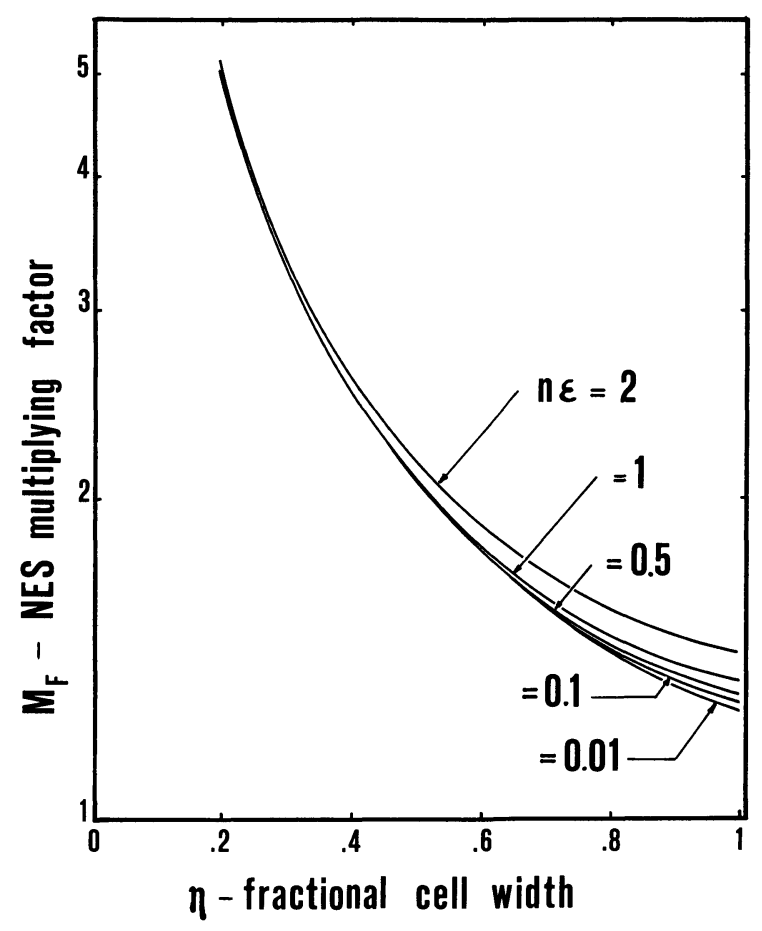

FIG. 9. - Multiplicative factor $M_{\mathrm{F}}$ of the $N E S$ in figure 8 when $N E S$ is referred to the actual CCD-input, as a function of fractional cell-width $\eta$.

Acknowledgment. - Thank are due to V. Svelto for constant encouragment. This work is sponsored by CNR-CCTE.

\section{References}

[1] Boyle, W. S., Smith, G. E., Bell Syst. Tech. J. 49 (1970) 587.

[2] Amelio, G. F., Tompsett, M. F., Sмith, G. E., Bell Syst. Tech. J. 49 (1970) 593.

[3] Carnes, J. E., Kosonocky, W. F., Ramberg, E. G., IEEE Trans. Electron Devices ED-19 (1972) 798.

[4] Buss, D. D., Bailey, W. H., Eversole, W. L., IEEE Trans. Electron Devices ED-22 (1975) 977.

[5] Donati, S., Svelto, V., IEEE Trans. Electron Devices ED-24 (1977) 1184.

[6] Joyce, W. B., Bertram, W. J., Bell Syst. Tech. J. 50 (1971) 1741.

[7] Barbe, D. F., Proc. IEEE 63 (1975) 38

[8] Chamberlain, S. G., Harper, D. H., IEEE Trans. Electron Devices ED-25 (1978) 145.
[9] Sequin, C. H., Tompsett, M. F., Charge Transfer Devices (Academic Press, New York) 1975.

[10] Berger, J. L., Coutures, J. L., IEEE J. Solid State Circuits SC-12 (1977) 617.

[11] Sequin, C. H., IEEE J. Solid State Circuits SC-12 (1977) 609.

[12] ThORNBeR, K. K., TOMPSETT, M. F., IEEE Trans. Electron Devices ED-20 (1973) 456.

[13] Donati, S., Gatri, E., Svelto, V., Adv. Electron. Electron Phys. (ed. by Morton) 26 (1969) 251.

[14] WEN, D. D., Low light level performances of CCD image sensors, in : Proc. of Int. Conf. on Appl. of CCD, oct. 29-31, 1975 . 